\title{
Incidencia de eventos cardiovasculares mayores en pacientes con infarto agudo del miocardio manejados con stent y su relación con la fracción de eyección ventricular
}

\author{
Sergio Morales ${ }^{1}$, Julián Ochoa ${ }^{2}$, Jorge Velasquez ${ }^{2}$. \\ Fundación Valle del Lili \\ Unidad de Cardiología Intervencionista. \\ Centro de Investigaciones Clínicas \\ 1 Residente Medicina Interna, convenio Universidad CES; 2 Especialista en Hemodinamia \\ El registro institucional DREST está financiado por el Centro de Investigaciones Clínicas de la \\ Fundación Valle del Lili y apoyado por la universidad ICESI.
}

Introducción: Los pacientes con infarto agudo del miocardio y disfunción ventricular izquierda tienen mayor riesgo de eventos cardiovasculares adversos mayores (MACE) y el uso de stent convencional vs fármaco-activo en este subgrupo de pacientes genera controversia.

Objetivos: Comparar la efectividad de los tipos de stent en pacientes con infarto agudo del miocardio y evaluar el impacto de la fracción de eyección ventricular en términos de MACE.

Métodos: Cohorte prospectiva basada en un registro de una institución de IV nivel de intervención percutánea coronaria con stent convencional o fármaco-activo. Se comparó la incidencia de MACE en pacientes con infarto agudo del miocardio según el tipo de stent en el primer año del evento y se evaluó el efecto de la fracción de eyección en la ocurrencia de desenlaces
Resultados: Se incluyeron 1084 pacientes con infarto agudo del miocardio entre enero 2002 hasta octubre de 2013 de los cuales $70,12 \%$ (758 pacientes) recibieron stent convencional (SC) y $29,88 \%$ stent fármaco-activo (SM). En un modelo multivariado no se observó beneficio del SM vs SC (HR 0,75 95\% IC 0,54-1,05 $\mathrm{p}=0,102$ ) respecto a presentar MACE, sin embargo la presencia de hipertensión arterial y disfunción ventricular fueron predictores independiente de presentar MACE (HR 1,51 95\% IC 1,15-2,0 p=0,003 y HR 1,61 95\% IC 1,22-2,13 p=0,001 respectivamente). Conclusiones: El uso de stent fármaco-activo no se relacionó con menor probabilidad de eventos adversos cardiovasculares mayores en pacientes con IAM. La disfunción ventricular izquierda fue un predictor de MACE a 1 año de seguimiento. 


\section{Incidence of major cardiovascular events in patients undergoing primary PTCA for Acute Myocardial Infarction: effect of stent type and left ventricular dysfunction at one year of follow up}

Background: Acute myocardial infarction and left ventricular dysfunction carry an increased risk of major adverse cardiovascular events ( MACE) and drug eluting stent used in this subgroup of patients is controversial.

Objectives: To compare the effectiveness of drug eluting stent (DES) vs bare metal stents (BMS) in patients with acute myocardial infarction (AMI). and to assess the impact of left ventricular ejection fraction on the incidence of MACE.

Methods: Data on a prospective cohort study of acute myocardial undergoing percutaneous coronary intervention with DES or BMS stents in a high complexity institution was analyzed. The incidence of MACE in the first year of follow-up and the effect of ejection fraction was assessed in relation to stent type and presence of left ventricular dysfunction Results: There were 1084 patients with AMI from January 2002 to October 2013. 70.12\% (758 patients) had BMS and $29.88 \%$ DES. In a multivariate analysis there was no benefit of DES vs BMS (HR $0.7595 \%$ CI 0.54 to $1.05 \mathrm{p}=0.102$ ). However, hypertension and ventricular dysfunction were independent predictors of MACE (HR $1.5195 \%$ CI 1.15 to $2.0 \mathrm{p}=0.003$ and HR $1.6195 \%$ CI 1.22 to $2.13 \mathrm{p}=0.001$ respectively). Conclusions: The use of DES was not associated with lower risk of MACE in patients with AMI. Left ventricular dysfunction was a predictor of MACE in the first year of follow up.

Keywords Drug-Eluting Stent; Ventricular dysfunction, Left; Myocardial Infarction 


\section{Introducción}

El infarto agudo de miocardio (IAM) es una de las principales causas de mortalidad a nivel mundial ${ }^{1}$. El pronóstico en los pacientes con IAM ha mejorado en las últimas dos décadas debido a los avances en el manejo farmacológico, la terapia de reperfusión con fibrinolíticos y la intervención percutánea coronaria primaria $(\mathrm{PCI})^{2-3}$. Esta última se ha asociado con una tasa más alta de permeabilidad de la arteria relacionada con el infarto, reestenosis menos severa, menor disfunción ventricular izquierda y tasa de isquemia recurrente e infarto, comparado con fibrinólisis ${ }^{4-5}$. En pacientes con IAM se han comparado los stent convencionales (SC) y fármaco-activos (SM) mostrando beneficios de este último grupo en cuanto a eventos adversos cardiovasculares mayores (MACE por sus siglas en inglés) sin encontrarse diferencias significativas en términos de mortalidad ${ }^{6-9}$. La disfunción ventricular izquierda en pacientes con IAM ha mostrado ser un fuerte predictor de mortalidad a 30 días y 1 año ${ }^{10}$. En la actualidad hay controversia respecto a la evolución de los pacientes con IAM manejados con SC y SM cuando se asocia con disfunción ventricular izquierda.

Se determinó la ocurrencia de MACE durante el primer año post-implante de SC o SM en pacientes con IAM sometidos a PCI y el efecto de la fracción de eyección en los desenlaces adversos cardiovasculares mayores (MACE).

\section{Métodos}

Se realizó un estudio de cohorte prospectivo basado en un registro sistemático (DREST) realizado desde 1994 en una institución de IV nivel de atención en Colombia que incluye todos los pacientes sometidos a intervención percutánea coronaria con stent convencional o fármaco-activo. A estos pacientes se les registró datos demográficos, información clínica relacionada con el procedimiento y seguimiento por vía telefónica, visita hospitalaria o con revisión de la historia clínica al egreso, al mes, 6 meses y al año hasta 5 años. Se incluyeron los pacientes mayores de 18 años, con indicación de implante de SC o SM por infarto agudo del miocardio con o sin ST elevado entre enero de 2002 y octubre de 2013 y se realizó seguimiento hasta el primer año después del implante del stent. Se definió como ocurrencia de MACE el combinado de muerte por cualquier causa, reinfarto, angina inestable que requirió hospitalización, trombosis aguda o reestenosis del stent, necesidad de repetir revascularización. La exposición principal fue el tipo de stent implantado y la co-variable principal fue la presencia de disfunción ventricular izquierda menor o igual a $45 \%$ por ventriculografía al momento del cateterismo cardíaco, se consideraron otras co-variables como los antecedentes personales, farmacológicos y hallazgos relevantes del procedimiento índice. Se comparó la ocurrencia de MACE en pacientes con IAM según el tipo de stent implantado y la presencia de disfunción ventricular. Las variables cualitativas se presentaron como número absoluto y porcentajes y las cuantitativas como medianas y rango intercuartílico. Se obtuvo un modelo multivariado de riesgos proporcionales Cox donde se incluyeron las variables estadísticamente significativas en el modelo univariado. Se estimó la probabilidad de estar libre de MACE hasta 1 año después del procedimiento y se compararon las sobrevidas mediante Log Rank Test. Se consideraron significativas $\mathrm{p}$ inferiores a 0,05. Se utilizó Stata 11.1 para realizar los análisis estadísticos. Este estudio fue aprobado por el Comité de Ética e Investigación Biomédica Institucional.

\section{Resultados}

Se incluyeron 1081 pacientes con infarto agudo del miocardio de los cuales 758 (70\%) recibieron stent convencional y $323(29,97 \%)$ stent fármaco-activo. La mediana de edad fue de 62 años en ambos grupos $(p=0,65)$ con una mayor prevalencia de hombres $(72,3 \%$ y $75,54 \%$ en SC y SM respectivamente). $56 \%$ de los pacientes en ambos grupos no fueron fumadores y se observó tabaquismo previo $y$ actual en $21 \%$ de los casos $(\mathrm{p}=0,953)$. La prevalencia de diabetes mellitus fue mayor en el grupo de stent fárma-

\begin{tabular}{|c|c|c|c|}
\hline \multicolumn{4}{|c|}{$\begin{array}{c}\text { Tabla 1. Características demográficas y antecedentes } \\
\text { de los pacientes con infarto agudo del miocardio según } \\
\text { tipo de stent implantado. }\end{array}$} \\
\hline Variable & $\begin{array}{c}\text { Stent } \\
\text { convencional } \\
\text { (758) }\end{array}$ & $\begin{array}{c}\text { Stent } \\
\text { fármaco-activo } \\
(n=323)\end{array}$ & $\mathrm{P}$ \\
\hline Edad & $62(53-70)$ & $62(54-70)$ & 0,65 \\
\hline Sexo masculino & $548(72,3)$ & $244(75,54)$ & 0,27 \\
\hline Diabetes mellitus & $153(20,18)$ & $84(26,01)$ & 0,03 \\
\hline Dislipidemia & $218(28,76)$ & $108(33,44)$ & 0,13 \\
\hline Hipertensión & $434(57,26)$ & $172(53,25)$ & 0,23 \\
\hline Infarto previo & $89(11,74)$ & $57(17,65)$ & 0,01 \\
\hline PTCA previo & $25(3,3)$ & $34(10,63)$ & 0,00 \\
\hline \multicolumn{4}{|l|}{ Farmacológicos } \\
\hline ASA & $355(46,83$ & ) $164(50,77)$ & 0,24 \\
\hline IECA & $206(27,18)$ & $75(23,22)$ & 0,18 \\
\hline ARA II & $91(12,01)$ & $67(20,74)$ & 0,00 \\
\hline Betabloqueadores & $206(27,18)$ & $117(36,22)$ & 0,00 \\
\hline
\end{tabular}

*Mediana (rango intercuartílico)

PTCA: angioplastía coronaria transluminal percutánea; ASA: Ácido acetil salicílico, ARA II: Antagonista del receptor de angiotensina. 
co-activo $(26,01 \%$ vs $20,18 \%$ p $=0,034)$. En la tabla 1 se describen las características clínicas principales según el tipo de stent. No hubo diferencias en el uso de medicamentos como clopidogrel, calcioantagonistas, nitratos, estatinas, heparinas de bajo peso molecular entre ambos grupos. Sin embargo, hubo mayor uso de antagonistas del receptor de angiotensina (ARA II) y betabloqueadores entre los pacientes que recibieron stent fármaco-activo (Tabla 1). La mediana de fracción de eyección ventricular por ventriculografía fue de $50 \%$ en ambos grupos. No hubo diferencias en el número de vasos enfermos en ambos grupos de SC y SM así como tampoco en el uso de inhibidores de glicoproteína IIb/IIIa. Fueron tratadas de forma más frecuente con SM cuando se presentaron dos o más lesiones comparado con $\mathrm{SC}(\mathrm{p}=0,00)$ (Tabla 2). En un modelo de riesgos proporcionales multivariado de Cox, luego de un año de seguimiento el uso de stent fármaco-activo en pacientes infarto agudo del miocardio no estuvo relacionado con

\begin{tabular}{|c|c|c|c|}
\hline \multicolumn{4}{|c|}{$\begin{array}{l}\text { Tabla 2. Hallazgos durante el procedimiento en } \\
\text { pacientes con infarto agudo del miocardio según el } \\
\text { tipo de stent implantado. }\end{array}$} \\
\hline Variable & $\begin{array}{l}\text { Stent } \\
\text { convencional } \\
(n=758)\end{array}$ & $\begin{array}{c}\text { Stent } \\
\text { fármaco- } \\
\text { activo }(n=323)\end{array}$ & $p$ \\
\hline \multicolumn{4}{|l|}{ Fracción } \\
\hline $\begin{array}{l}\text { eyección } \\
\text { Número va }\end{array}$ & $50(40-55)$ & $50(45-55)$ & 0,00 \\
\hline 1 & $374(49,54)$ & $166(51,55)$ & 0,77 \\
\hline 2 & $227(30,07)$ & $96(29,81)$ & \\
\hline 3 & $154(20,40)$ & $60(18,63)$ & \\
\hline \multicolumn{4}{|c|}{ Inhibidores GP IIbllla } \\
\hline & $281(37,07)$ & $111 \quad(34,37$ & 0,40 \\
\hline \multicolumn{4}{|c|}{ No Lesiones tratadas } \\
\hline 1 & $678(89,45)$ & $260(80,50)$ & 0,00 \\
\hline 2 & $73(9,63)$ & $58(17,96)$ & \\
\hline 3 & $7(0,92)$ & $5(1,55)$ & \\
\hline \multicolumn{4}{|c|}{ Longitud de la lesión } \\
\hline & $18(13-22)$ & $20(14-28)$ & 0,00 \\
\hline \multicolumn{4}{|l|}{ Flujo TIMI } \\
\hline 0 & $282(37,55)$ & $67(20,94)$ & 0,00 \\
\hline 1 & $19(2,53)$ & $6(1,88)$ & \\
\hline 2 & $65(8,66)$ & $19(5,94)$ & \\
\hline 3 & $385(51,26)$ & $228(71,25)$ & \\
\hline \multicolumn{4}{|c|}{ Presencia de trombo } \\
\hline & $291(38,44)$ & $70(21,67)$ & 0,00 \\
\hline \multicolumn{4}{|c|}{ Reestenosis intrastent } \\
\hline & $6(0,79)$ & $8(2,48)$ & 0,03 \\
\hline \multicolumn{4}{|c|}{ No. De stents } \\
\hline 1 & $623(82,19$ & $235(72,76)$ & 0,00 \\
\hline 2 & $135(17,81)$ & $88(27,24)$ & \\
\hline
\end{tabular}

mayor o menor incidencia de MACE (HR 0,75 95\% IC 0,54-1,05 p=0,102). La presencia de disfunción ventricular izquierda menor a $45 \%$ se asoció con $60 \%$ más riesgo de presentar MACE independiente de la edad, presencia de diabetes, hipertensión arterial y hallazgos angiográficos. La hipertensión arterial se relacionó con 50\% mayor probabilidad de presentar MACE a 1 año de seguimiento (Tabla 3, Figura 1, 2 y 3 ).

\section{Discusión}

En los pacientes con infarto agudo de miocardio, los estudios que comparan el uso de SC y SM han mostrado que los segundos se asocian con un porcentaje menor de re-es-

Tabla 3. Modelo de riesgos proporcionales Cox con seguimiento a 1 año y desenlace adverso MACE

\begin{tabular}{|l|r|r|r|}
\hline Variable & HR & $95 \%$ IC & P \\
\hline Stent fármaco-activo & 0,75 & $0,54-1,05$ & 0,102 \\
\hline Diabetes Mellitus & 1,12 & $0,82-1,52$ & 0,461 \\
\hline Hipertensión & 1,51 & $1,15-2,0$ & 0,003 \\
\hline Disfunción ventricular & 1,61 & $1,22-2,13$ & 0,001 \\
\hline Inh Gpllbllla & 1,11 & $0,80-1,55$ & 0,505 \\
\hline No Lesiones tratadas & 1,01 & $0,71-1,44$ & 0,935 \\
\hline Estenosis inicial & 1 & $0,98-1,02$ & 0,717 \\
\hline Lesión ostial & 0,87 & $0,50-1,5$ & 0,628 \\
\hline Trombo presente & 0,93 & $0,66-1,32$ & 0,717 \\
\hline Reestenosis intrastent & 0,76 & $0,23-2,42$ & 0,646 \\
\hline
\end{tabular}

Figura 1. Diagrama de cajas y bigotes de distribución de fracción de eyección ventricular según el tipo de stent y la presencia de desenlaces cardiovasculares adversos mayores.

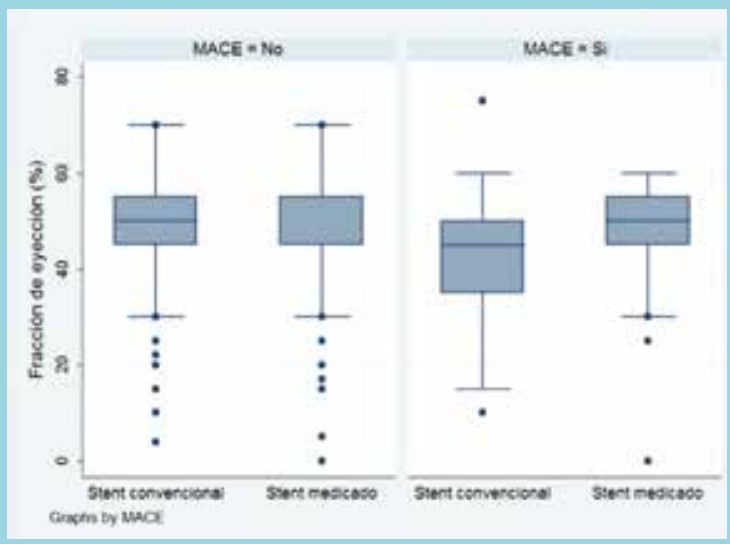

MACE: Evento adverso cardiovascular mayor 

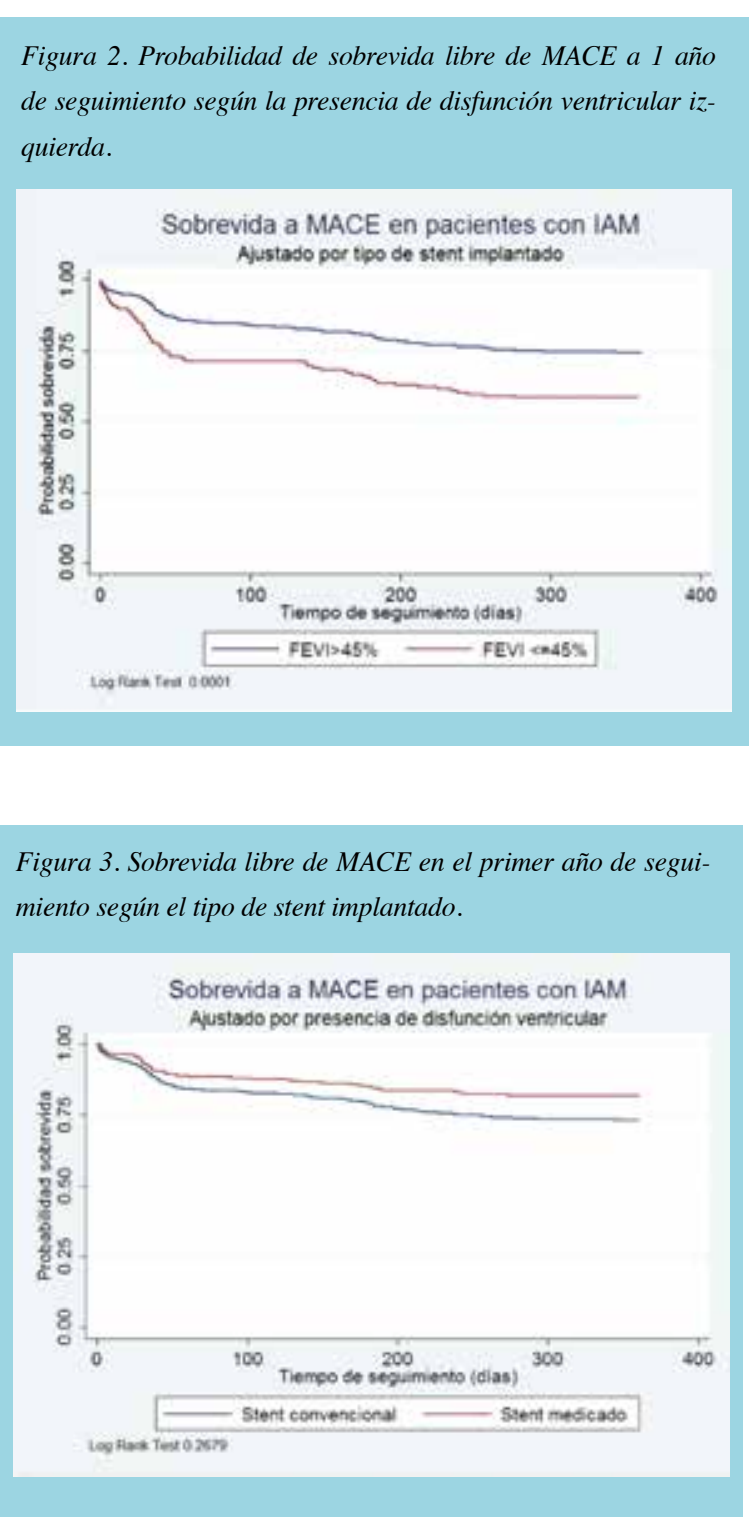

tenosis y una menor necesidad de revascularizar el vaso afectado, sin embargo, con mayor riesgo de trombosis tardía asociado con el uso de stent fármaco-activo ${ }^{6-11-20}$. En nuestro estudio, se encontró menor frecuencia de eventos en los que recibieron SM vs SC, sin ser esta diferencia significativa a 1 año de seguimiento. No se observó mayor frecuencia de trombosis tardía del stent. También se observó mayor probabilidad de presentar MACE cuando hay disfunción ventricular izquierda y que por cada $1 \%$ de aumento de la función ventricular la probabilidad de presentar MACE disminuía 3\% a un año de seguimiento (HR 0,97 95\% IC 0,96-0,98 p=0,000). En estudios de pacientes con IAM a quienes se realiza una angioplastía primaria con implantación de stent, la disfunción ventricular ha mostrado ser un predictor importante de mortalidad a 30 días y a 1 año ${ }^{10-21}$, además de estar relacionada con trombosis tardía del stent ${ }^{22}$. Sin embargo, en pacientes con lesión de tronco que fueron intervenidos y se les implantó stent, la disfunción ventricular izquierda no mostró ser un factor pronóstico independiente después del análisis multivariado $^{23}$. Niveles de NT-proBNP mayores de 463,4 pg/ $\mathrm{ml}$ en pacientes con IAM e implantación de stent permiten identificar de manera temprana pacientes con disfunción ventricular, lo cual es importante vista la morbimortalidad de este grupo ${ }^{24}$. La principal limitante de este es que los pacientes no fueron aleatorizados para recibir SC o SM. Nuestros resultados pueden estar afectados por un sesgo de selección con respecto a las características de severidad de las lesiones que fueron diferentes en ambos grupos. Debido a que este es un estudio basado en los resultados de un registro institucional, la probabilidad de recibir uno $\mathrm{u}$ otro stent según el perfil clínico del paciente pudo sesgar los resultados ${ }^{25}$. Además, se encontró una diferencia cronológica en la implantación de los stent, siendo los SC los más usados al principio del estudio y los SM predominantes en los últimos años lo cual puede relacionarse no sólo con las características del stent si no con más experiencia de los operadores, mejores técnicas y mejor manejo médico. Aunque se realizaron análisis multivariados para minimizar el efecto de las diferencias encontradas en las características de base registradas puede seguir existiendo efecto de factores confundentes no identificados en este estudio. A pesar de esto, consideramos que la medición de desenlaces duros no relacionados con la decisión del tipo de stent implantado permite describir estos eventos.

\section{Conclusiones}

La ocurrencia de MACE en pacientes llevados a implantación de stent coronario por IAM no se relacionó con el tipo de stent implantado. Sin embargo, la presencia de disfunción ventricular izquierda se asoció con mayor probabilidad de desenlaces adversos. 
1. MURRAY CJ, LÓPEZ AD. Alternative projections of mortality and disability by cause 1990-2020: Global Burden of Disease Study. Lancet 1997; 349: 1498-504.

2. COLLINS R, PETO R, BAIGENT C, SLEIGHT P. Aspirin, heparin, and fibrinolytic therapy in suspected acute myocardial infarction. N Engl J Med. 1997; 336: 847-60.

3. HENNEKENS CH, ALBERT CM, GODFRIED SL, GAZIANO JM, BURING JE. Adjunctive drug therapy of acute myocardial infarction--evidence from clinical trials. N Engl J Med 1996; 335: 1660-8.

4. ZIJLSTRA F, DE BOER MJ, HOORNTJE JC, REIFFERS S, REIBER JH, SURYAPRANATA H. A comparison of immediate coronary angioplasty with intravenous streptokinase in acute myocardial infarction. N Engl J Med 1993; 328: 680-4.

5. KEELEY EC, BOURA JA, GRINES CL. Primary angioplasty versus intravenous thrombolytic therapy for acute myocardial infarction: a quantitative review of 23 randomised trials. Lancet 2003; 361: 13-20.

6. DI LORENZO E, DE LUCA G, SAURO R, VARRICCHIO A, CAPASSO M, LANZILLO T, et al. The PASEO (PaclitAxel or Sirolimus-Eluting Stent Versus Bare Metal Stent in Primary Angioplasty) Randomized Trial. JACC Cardiovasc interv. 2009; 2:515-23.

7. HAO P-P, CHEN Y-G, WANG X-L, ZHANG Y. Efficacy and safety of drug-eluting stents in patients with acute ST-segment-elevation myocardial infarction: a meta-analysis of randomized controlled trials. Tex Heart Inst J. 2010; 37: 516-24.

8. KALESAN B, PILGRIM T, HEINIMANN K, RÄBER L, STEFANINI GG, VALGIMIGLI M, et al. Comparison of drug-eluting stents with bare metal stents in patients with ST segment elevation myocardial infarction. Eur Heart J 2012; 33: 977-87.

9. STEG PG, FOX KAA, EAGLE KA, FURMAN M, VAN DE WERF F, MONTALESCOT G, et al. Mortality following placement of drug-eluting and bare-metal stents for ST-segment elevation acute myocardial infarction in the Global Registry of Acute Coronary Events. Eur Heart J. 2009; 30: 321-9.

10. RASOUL S, OTTERVANGER JP, DE BOER M-J, DAMBRINK J-HE, HOORNTJE JCA, MARCEL GOSSELINK AT, et al. Predictors of 30-day and 1-year mortality after primary percutaneous coronary intervention for ST-elevation myocardial infarction. Coron Artery Dis 2009; 20: 415-21.

11. MOSES JW, LEON MB, POPMA JJ, FITZGERALD PJ, HOLMES DR, O"SHAUGHNESSY C, et al. Sirolimus-eluting stents versus standard stents in patients with stenosis in a native coronary artery. N Engl J Med 2003; 349: 1315-23.

12. ROIRON C, SANCHEZ P, BOUZAMONDO A, LECHAT P, MONTALESCOT G. Drug eluting stents: an updated meta-analysis of randomised controlled trials. Heart. 2006; 92: $641-9$.

13. STONE GW, ELLIS SG, CANNON L, MANN JT, GREENBERG JD, SPRIGGS D, et al. Comparison of a polymer-based paclitaxel-eluting stent with a bare metal stent in patients with complex coronary artery disease: a randomized controlled trial. JAMA 2005; 294: 1215-23.

14. ARDISSINO D, CAVALLINI C, BRAMUCCI E, INDOLFI C, MARZOCCHI A, MANARI A, et al. Sirolimus-eluting vs uncoated stents for prevention of restenosis in small coronary arteries: a randomized trial. JAMA 2004; 292: 2727-34.

15. MCFADDEN EP, STABILE E, REGAR E, CHENEAU E, ONG ATL, KINNAIRD T, et al. Late thrombosis in drug-eluting coronary stents after discontinuation of antiplatelet therapy. Lan

16. MARCOLINO MS, SIMSEK C, DE BOER SPM, VAN DOMBURG RT, VAN GEUNS R-J, DE JAEGERE P, et al. Shortand long-term major adverse cardiac events in patients undergoing percutaneous coronary intervention with stenting for acute myocardial infarction complicated by cardiogenic shock. Cardiology 2012; 121: 47-55.

17. LAGERQVIST B, JAMES SK, STENESTRAND U, LIND- 
BÄCK J, NILSSON T, WALLENTIN L. Longterm outcomes with drug-eluting stents versus bare-metal stents in Sweden. N Engl J Med 2007; 356: 1009-19.

18. IAKOVOU I, SCHMIDT T, BONIZZONI E, GE L, SANGIORGI GM, STANKOVIC G, et al. Incidence, predictors, and outcome of thrombosis after successful implantation of drug-eluting stents. JAMA 2005; 293: 2126-30.

19. NAKAZAWA G, FINN AV, JONER M, LADICH E, KUTYS $\mathrm{R}, \mathrm{MONT}$ EK, et al. Delayed arterial healing and increased late stent thrombosis at culprit sites after drug-eluting stent placement for acute myocardial infarction patients: an autopsy study. Circulation 2008; 118: 1138-45.

20. MIERES J, RODRÍGUEZ AE. Stent Selection in Patients with Myocardial Infarction: Drug Eluting, Biodegradable Polymers or Bare Metal Stents? Recent pat Cardiovasc Drug Discov. 2012; 7: 105-120.

21. SHAW LJ, MIERES JH, HENDEL RH, BODEN WE, GULATI M, VELEDAR E, et al. Comparative effectiveness of exercise electrocardiography with or without myocardial perfusion single photon emission computed tomography in women with suspected coronary artery disease: results from the What Is the
Optimal Method for Ischemia Evaluation. Circulation 2011; 124:1239-49.

22. HALKIN A, STONE GW, DIXON SR, GRINES CL, TCHENG JE, COX DA, et al. Impact and determinants of left ventricular function in patients undergoing primary percutaneous coronary intervention in acute myocardial infarction. Am J Cardiol 2005; 96: $325-31$.

23. JIN C, LU L, ZHU ZB, ZHANG RY, ZHANG Q, DU R, et al. Increased serum vWF and SVCAM-1 levels are associated with late or very late angiographic stent thrombosis after sirolimus-eluting stent implantation. Coron Artery Dis. 2010; 21: 273-7.

24. BIONDI-ZOCCAI G, SHEIBAN I, MORETTI C, PALMERINI T, MARZOCCHI A, CAPODANNO D, et al. Appraising the impact of left ventricular ejection fraction on outcomes of percutaneous drug-eluting stenting for unprotected left main disease: insights from a multicenter registry of 975 patients. Clin Res Cardiol. 2011; 100: 403-11.

25. FLAHERTY JD, DAVIDSON CJ, FAXON DP. Percutaneous coronary intervention for myocardial infarction with left ventricular dysfunction. Am J Cardiol 2008; 102: 38G-41G. 